\title{
Acoustic attenuation in a type-II superconductor at high magnetic fields
}

\author{
G. M. Bruun, V. Nikos Nicopoulos, N. F. Johnson \\ Department of Physics, Clarendon Laboratory, University of Oxford, Oxford OX1 3PU, England
}

(October 22, 2018)

\begin{abstract}
We have calculated the longitudinal acoustic attenuation in a type II superconductor in high magnetic fields within a mean-field BCS theory. We predict two new features in the corresponding attenuation signal as compared to that of the Meissner state. Our analytical calculations predict the existence of oscillations in the attenuation as the external magnetic field is varied- this effect is associated with the Landau level structure of the electron states and is analogous to the well-known de Haas van Alphen oscillations in the mixed state. The attenuation directly probes the quasiparticle energies; the presence of gapless points in the quasiparticle spectrum, which is characteristic of type-II superconductors at high magnetic fields, shows up in the frequency $\omega$ and temperature $T$ dependence of the attenuation in the limit of low $\omega$ and and low $T$ respectively. At low $T$ there is no analogue to the discontinuity in the attenuation observed in the Meissner state when $\hbar \omega=2 \Delta$, where $\Delta$ is the quasiparticle energy gap. This result opens up the possiblity of experimentally determining the existence and nature of the gapless points in the quasiparticle spectrum of a type-II superconductor in high magnetic fields.
\end{abstract}

PACS numbers: 74.25.Ld, 74.60.-w

\section{INTRODUCTION}

The problem of understanding type-II superconductivity in high magnetic fields has been a lively topic for some years now. When $k_{B} T<\hbar \omega_{c}$, where $\omega_{c}=e H / m c$ is the cyclotron frequency, the Landau level quantization of the electron levels become important. This gives rise to a number of interesting effects. 1 One such effect is the existence of magnetic oscillations in the free energy of the superconductor in the mixed state. These de Haas-van Alphen ( $\mathrm{dHvA}$ ) oscillations was observed over 20 years agd 3 and have since been observed in a number of materials. One important theoretical consequence of the Landau level quantization is the presence of gapless points in the quasiparticle spectrum. Theoretical investigations have shown 6 that the quasiparticle spectrum of the mixed state in high magnetic fields is characterized by a set of gapless points in the magnetic Brillouin zone (MBZ). These gapless point leads to an algebraic temperature dependence of the thermodynamic functions and an algebraic voltage dependence in the tunneling conductance. 1 It has been suggested $\mathbf{b}$ that the existence of the dHvA oscillations in the mixed state is a consequence of these gapless points. It turns out however, that it is rather difficult to develop a simple, yet consistent theory for the dHvA-oscillations since both the oscillatory behaviour of the ground state energy and the gapless nature of the quasiparticle energies need to be taken into account 6 The interpretation of the experimental results for the dHvA-oscillations is consequently somewhat unclear and does not, in our opinion, give a completely unambiguous signature for the presence of gapless modes.

In this paper we consider the attenuation of longitudinal acoustic waves in the mixed state where the order parameter is assumed to form a vortex lattice. Since the absorption of the phonons is due to quasiparticle excitations, the experiment directly probes the quasiparticle density of states. For clean materials, theoretical results have proved rather difficult to obtain since an expansion in powers of the order parameter does not converget and the semiclassical approximation leads to unphysical results. Since we consider the superconductor at high magnetic fields, it is crucial to take into account the Landau level quantization of the electronic levels. It turns out that by including this effect, one avoids the difficulties encountered in the semiclassical approximation as predicted by Scharnberg. We calculate analytically the attenuation in two limits: $k_{B} T \ll \hbar \omega$ and $k_{B} T \gg \hbar \omega$ where $\omega$ is the frequency of the sound wave and $T$ is the temperature. We predict that the attenuation will be an oscillatory function of the external magnetic field due to the Landau level structure, in analogy with the usual dHvA oscillations. The frequency and temperature dependence of the attenuation is determined by the existence and the nature of the gapless points in the quasiparticle spectrum for $k_{B} T \ll \hbar \omega$ and $k_{B} T \gg \hbar \omega$ respectively. This in principle gives an experimental tool for probing the nature of the quasiparticle energies in the mixed state. Our analytical theory is supported by essentially exact numerical calculations for the attenuation.

As the analysis in this article will show, the existence of the gapless points in the quasiparticle spectrum has significant consequences on the longitudinal sound attenuation. We would therefore expect that this should also hold for systems with intrinsic gapless points even for no external field such as high-temperature superconductors (high- $T_{c}$ 's), which seems to have a $d$-wave gap symmetry. Hence a similar analysis of the acoustic attenuation in 
these high- $T_{c}$ 's as the one presented in this paper for type-II superconductors in high magnetic fields would be very interesting, and we will return to this in a future publication.

\section{GENERAL THEORY}

We consider a weak-coupling superconductor in three dimensions (3D) described within mean field theory by the following Hamiltonian:

$$
\hat{H}=\sum_{\sigma} \int d \mathbf{r} \psi_{\sigma}^{\dagger}(\mathbf{r})\left(\frac{\left(\mathbf{p}-\frac{e}{c} \mathbf{A}\right)^{2}}{2 m}-\mu\right) \psi_{\sigma}(\mathbf{r})+\int d \mathbf{r}\left[\Delta(\mathbf{r}) \psi_{\uparrow}^{\dagger}(\mathbf{r}) \psi_{\downarrow}^{\dagger}(\mathbf{r})+c . c\right]
$$

where the order parameter is defined as $\Delta(\mathbf{r})=g\left\langle\psi_{\uparrow}(\mathbf{r}) \psi_{\downarrow}(\mathbf{r})\right\rangle, g$ is the coupling strength and $\mu$ is the chemical potential. Our theory describes longitudinal ultrasonic waves in clean samples for high magnetic fields. We have for simplicity confined our theory to the case when $\mathbf{q} \| \mathbf{H}$ where $\mathbf{q}$ is the wavevector of the sound wave and $\mathbf{H}$ is the magnetic field. This simplifies our calculations since the coupling between the collective modes associated with fluctuations of the order parameter and the longitudinal phonons can be neglected to a good approximation. 010 The attenuation of sound waves is given by the imaginary partgf the retarded phonon self energy. To lowest order in the electron-phonon coupling we get for the attenuation $\alpha(\omega) 11$

$$
\alpha(\omega) \propto-\omega \operatorname{Im}\left\{D^{R}(\mathbf{q}, \omega)\right\}
$$

where $D^{R}(\mathbf{q}, \omega)$ is the retarded density-density correlation function

$$
i D^{R}\left(\mathbf{x}, \mathbf{x}^{\prime}, t-t^{\prime}\right)=\left\langle\left[\tilde{n}(\mathbf{x}, t), \tilde{n}\left(\mathbf{x}^{\prime}, t^{\prime}\right)\right]\right\rangle \theta\left(t-t^{\prime}\right)
$$

and $\tilde{n}(\mathbf{x}, t)=\hat{n}(\mathbf{x}, t)-\langle\hat{n}(\mathbf{x}, t)\rangle$ is the operator describing density fluctuations. This function is evaluated as the analytical continuation of the thermal correlation function. We will in this paper for simplicity ignore the electronic Zeeman effect. The effect of a fnite spin splitting on the magnetic oscillations is well understood both in the normal state 12 and in the mixed state. Hence ignoring the spin splitting we obtain

$$
\begin{array}{r}
D^{R}(\mathbf{q}, \omega)=\frac{2 k_{B} T}{V_{\text {cell }}} \sum_{\omega_{\nu}} \int_{\text {cell }} d^{2} \mathbf{r} \int d^{3} \mathbf{r}^{\prime} e^{\mathbf{q \mathbf { r } ^ { \prime }}}\left[G\left(\mathbf{r}, \mathbf{r}^{\prime}, \omega_{\nu}\right) G\left(\mathbf{r}^{\prime}, \mathbf{r}, \omega_{\nu}-\omega_{\gamma}\right)\right. \\
\left.-F^{\dagger}\left(\mathbf{r}, \mathbf{r}^{\prime}, \omega_{\nu}\right) F\left(\mathbf{r}^{\prime}, \mathbf{r}, \omega_{\nu}-\omega_{\gamma}\right)\right]\left.\right|_{i \omega_{\gamma} \rightarrow \omega+i \delta}
\end{array}
$$

where $G\left(\mathbf{r}, \mathbf{r}^{\prime}, \omega_{\nu}\right)$ and $F\left(\mathbf{r}^{\prime}, \mathbf{r}, \omega_{\nu}\right)$ are the one-particle Green's functions for the superconductor. The symbol $\int_{\text {cell }} d^{2} \mathbf{r}$ implies integration over one vortex lattice cell in the $x-y$ plane whereas $\int d^{3} \mathbf{r}^{\prime}$ means integration over the whole crystal. By treating the electron-phonon matrix element as an overall factor in our formalism (i.e. its frequency dependence $\omega$ is included as a prefactor in Eq.(2)) we have made use of the fact that the screening for longitudinal modes is essentially the same as in the normal phase.13 Since the order parameter is assumed to form a vortex lattice, we can use a set of single particle states $\phi_{n \mathbf{k} k_{z}}(\mathbf{r})$ characterized by a Landau level index $n$, a wavevector $\mathbf{k}=\left(k_{x}, k_{y}\right)$ in the MBZ, and a wavevector $k_{z}$ along the $z$-direction. These basis states were introduced by Norman et al.3 In this basis there is no mixing between different k's since the sound wave travels along the $z$-direction. Expanding the Green's functions in this basis and using the $\mathbf{k}$-space symmetry of the problem due to the presence of the vortex lattice, 3 we obtain the following expression for the ultrasonic attenuation in the mixed state of a type-II superconductor:

$$
\begin{aligned}
\alpha(\mathbf{q}, \omega) \propto & \frac{\omega}{L_{x} L_{y}} \sum_{n n^{\prime}} \sum_{\mathbf{k}} \int d k_{z}\left\{[ f ( E ) - f ( E ^ { \prime } ) ] \left[\delta ( E ^ { \prime } - E - \omega ) \left(\left|U^{\prime} \wedge U\right|^{2}-\right.\right.\right. \\
& \left.\left.-V^{\prime} \wedge V^{*} U^{\prime *} \wedge U\right)-\delta\left(E-E^{\prime}-\omega\right)\left(\left|V^{\prime *} \wedge V\right|^{2}-U^{\prime *} \wedge U V^{\prime} \wedge V^{*}\right)\right] \\
& \left.+\left[1-f\left(E^{\prime}\right)-f(E)\right] \delta\left(E^{\prime}+E-\omega\right)\left(\left|U^{\prime} \wedge V\right|^{2}+V^{\prime} \wedge U U^{\prime *} \wedge V^{*}\right)\right\} .
\end{aligned}
$$

Here $U \equiv U_{\mathbf{k} k_{z}}^{n}(\mathbf{r}), V \equiv V_{\mathbf{k} k_{z}}^{n}(\mathbf{r}), U^{\prime} \equiv U_{\mathbf{k} k_{z}+q}^{n^{\prime}}(\mathbf{r})$ and $V^{\prime} \equiv V_{\mathbf{k} k_{z}+q}^{n^{\prime}}(\mathbf{r})$ are the Bogoliubov functions. The quasiparticle energies are given by $E \equiv E_{k_{z}}^{n}(\mathbf{k})$ and $E^{\prime} \equiv E_{k_{z}+q}^{n^{\prime}}(\mathbf{k}) ; f(E)=1 /\left(\exp \left(E / k_{B} T\right)+1\right)$ and the $\wedge$-product means integration over the $x y$-plane. The quasiparticle energies and associated wavefunctions can be found by solving the corresponding Bogoliubov-de Gennes (BdG) equations.14 We have set up a program that solves the BdG equations self-consistently in $3 \mathrm{D}$ such that we get the Bogoliubov functions as a function of $H$. The method for solving these 
equations numerically in $2 \mathrm{D}$ is described in detail elsewhere 1 The extension to $3 \mathrm{D}$ is straightforward although it is computationally significantly more demanding. We choose the normal state dispersion law along the $z$-direction to be either the plane wave form $\epsilon\left(k_{z}\right)=k_{z}^{2} / 2 m$, or more suitable for layered structures, the tight-binding form $\epsilon\left(k_{z}\right)=t \cos \left(k_{z} a_{z}\right)$ where $a_{z}$ is the distance between the planes.

In order to develop an analytical theory for the attenuation we need to make some approximations. Near the upper critical field $H_{c 2}$ and for quasiparticle levels close to the Fermi energy we can, as a first approximation, igpere the off-diagonal pairing (i.e. the so-called diagonal approximation). The quasiparticle energies are then given by 15

$$
E_{k_{z}}^{n}(\mathbf{k})=\sqrt{\xi_{n}\left(k_{z}\right)^{2}+|\Delta(\mathbf{k})|^{2}}
$$

and the corresponding Bogoliubov functions are given by

$$
\left.\begin{array}{l}
\left|U_{\mathbf{k} k_{z}}^{n}(\mathbf{r})\right|^{2} \\
\left|V_{\mathbf{k} k_{z}}^{n}(\mathbf{r})\right|^{2}
\end{array}\right\}=\frac{1}{2}\left(1 \pm \frac{\xi_{n}\left(k_{z}\right)}{E_{k_{z}}^{n}(\mathbf{k})}\right)\left|\phi_{n \mathbf{k} k_{z}}(\mathbf{r})\right|^{2} .
$$

Here $\xi_{n}\left(k_{z}\right)=n \hbar \omega_{c}+\epsilon\left(k_{z}\right)-\mu$. It should be noted that this approximation is only valid for energy levels close to the Fermi energy. Further away from the Fermi level there are degeneracies between electron and hole states belonging to different Landau levels, hence our approximation will eventually breake down. However, for low frequencies and temperatures one can show that only levels for which Eq.(6) and Eq.(7) hold will contribute to the damping described by Eq.(5). A closer examination shows the requirement for the diagonal approximation to hold to $\max \left(k_{B} T, \omega\right) \leqslant \omega_{c} / 4$. This follows because mixing with hole levels become important for levels with $E \geq \hbar \omega_{c} / 4.6 .16$ Dukan et al. 15 argued that the off-diagonal pairing does not change the qualitative behavior of the superconductor in a high magnetic field for fields not too far below $H_{c 2}$, and that the quasiparticle spectrum remains essentially the same when the off-diagonal terms are included. From the diagonal approximation, it directly follows that there are gapless points in the MBZ (i.e $\Delta(\mathbf{k})=0$ ). Even when the diagonal approximation breaks down, there will still be points in the MBZ where the gap vanishes. It seems the role of the off-diagonal terms is to shift the value of the Fermi momentum $k_{z f}$ where the gapless behaviour occurs away from its diagonal approximation value $\epsilon\left(k_{z f}\right)=0.0$ Eventually, true gapped behaviour sets in when the superconducting order is strong enough to increase the energies of the quasibound states in the vortices above $\hbar \omega_{c}$.

In Fig. 1 we have plotted the lowest quasiparticle energy, calculated numerically, along the $\Gamma-M$ direction in $\mathbf{k}$-space for two different values of the order parameter $\Delta(\mathbf{r})$ at a low temperature. The two points $\Gamma=\left(\pi / 4 a_{x}, \pi / 2 a_{y}\right)$ and $M=\left(3 \pi / 4 q_{x}, \pi / 2 a_{y}\right)$ are two of the corners in an irreducible triangle reflecting the symmetry of the BdG-equations in k-space 3 Here $a_{x}=l(\sqrt{3} \pi / 2)^{1 / 2}$ as we have chosen a triangular symmetry for the vortex lattice, $a_{y}=l^{2} / a_{x}$ and $l^{2}=\hbar c / e H$ is the magnetic length. We have chosen the size of the system such that $\pi /\left(2 a_{x} \Delta k_{x}\right)=50$ where $\Delta k_{x}=2 \pi / L x$ and $L_{x}$ is the extend of the system in the $x$-direction. The dashed lines give the diagonal approximation to the energies and the solid lines the exact numerical result. The two highest-lying curves are calculated with $\langle\Delta(\mathbf{k})\rangle \simeq 0.3 \hbar \omega_{c}$ and the two lowest lying curves are with $\langle\Delta(\mathbf{k})\rangle \simeq 0.05 \hbar \omega_{c}$. Here $\langle\Delta(\mathbf{k})\rangle$ means the $\mathbf{k}$-space average of the diagonal matrix element in the BdG equations. There are 10 Landau levels within the pairing width and the dispersion law along the z-direction is $\epsilon\left(k_{z}\right)=t \cos \left(k_{z} a_{z}\right)$. The value of $k_{z}$ is chosen such that $\epsilon\left(k_{z}\right)=0$. As can be seen, the diagonal approximation predicts two gapless points along this k-space direction, both with a linear dispersion law. For a small pairing parameter there is good agreement between the diagonal approximation and the full calculation. For a larger pairing parameter, i.e. $\langle\Delta(\mathbf{k})\rangle \simeq 0.3 \hbar \omega_{c}$, the approximation is less precise- indeed the gapless points predicted by the diagonal approximation disappear in the full self-consistent calculation. This is due to the off-diagonal pairing which become increasingly important as the pairing interaction increases deeper into the mixed state. The gapless points are now at a different value of $k_{z}$. We expect our theory to be valid reasonably close to the transition line such that we can ignore the off-diagonal pairing. As will be shown later, the major contribution actually comes from the gapless points where the diagonal approximation is most valid. We will now calculate the attenuation in two limits using this approximation.

\section{LOW FREQUENCY}

We will first treat the case $\omega \ll k_{B} T$. In this limit we can focus on the first two delta functions in Eq.(5) which describe the scattering of a quasiparticle. We will outline the calculation for the dispersion law $\epsilon\left(k_{z}\right)=k_{z}^{2} / 2 m$. Making the approximation $\xi^{\prime} / E^{\prime} \simeq \xi / E$ which is valid for $\hbar \omega \ll k_{B} T$ the first two terms of Eq.(可) become:

$$
\alpha(\mathbf{q}, \omega)=-\frac{\omega^{2} m}{\hbar L_{x} L_{y} q} \sum_{n} \sum_{\mathbf{k}} \int d k_{z} \delta\left(k_{z}-k_{z}^{*}(n, \mathbf{k})\right) \partial_{E} f\left(E_{k_{z}}^{n}(\mathbf{k})\right) \frac{\left|\xi_{n}\left(k_{z}\right)\right|}{E_{k_{z}}^{n}(\mathbf{k})}
$$


Here $k_{z}^{*}(n, \mathbf{k})$ is the solution to the equation $E_{k_{z}+q}^{n}(\mathbf{k})-E_{k_{z}}^{n}(\mathbf{k})=\omega$. From the Poisson formula we have the identity

$$
\sum_{n=-\infty}^{\infty} \partial_{E} f\left(E_{k_{z}^{*}}^{n}(\mathbf{k})\right) \frac{\left|\xi_{n}\left(k_{z}^{*}\right)\right|}{E_{k_{z}^{*}}^{n}(\mathbf{k})}=\sum_{j=-\infty}^{\infty} \int d x^{2 j \pi i x} \partial_{E} f\left(E_{k_{z}^{*}}^{x}(\mathbf{k})\right) \frac{\left|\xi_{x}\left(k_{z}^{*}\right)\right|}{E_{k_{z}^{*}}^{x}(\mathbf{k})}
$$

where $\xi_{x}\left(k_{z}^{*}\right)=x \hbar \omega_{c}+\epsilon\left(k_{z}^{*}\right)-\mu=\left(x-n_{f}\right) \hbar \omega_{c}+\epsilon\left(k_{z}^{*}\right)$. Here $n_{f} \equiv \mu / \hbar \omega_{c}-0.5$ is the Landau level index at the chemical potential. We have extended the Landau level sum to go from $-\infty$ as the low levels do not contribute anyway. Making the variable substitution $\xi_{x}\left(k_{z}^{*}\right)=z \hbar \omega_{c}$ we end up with

$$
\alpha(\mathbf{q}, \omega)=-\frac{\omega^{2} m}{\hbar L_{x} L_{y} q} \sum_{\mathbf{k}} \sum_{j=-\infty}^{\infty} e^{2 \pi i j\left(n_{f}-\epsilon\left(k_{z}^{*}\right) / \hbar \omega_{c}\right)} \int_{-\infty}^{\infty} d z e^{2 \pi i j z} \partial_{E} f\left(E_{z}(\mathbf{k})\right) \frac{\left|\xi_{z}\right|}{E_{z}(\mathbf{k})}
$$

where $E_{z}(\mathbf{k})=\left(\xi_{z}^{2}+\Delta(\mathbf{k})\right)^{1 / 2}$. We have approximated $k_{z}^{*}$ as the corresponding normal state solution (i.e. it is independent of $n$ ). This approximation which is only necessary when we calculate the oscillations of the attenuation (i.e. the terms in Eq.(10) with $j \neq 0$ ), should be good close to the gapless points and is equivalent to ignoring any phaseshift in the oscillations due to the superconducting order. The 0 'th harmonic is given by the $j=0$ term. We get

$$
\alpha(\mathbf{q}, \omega)_{0}=\frac{2 \omega^{2} m}{\hbar^{2} \omega_{c} L_{x} L_{y} q} \sum_{\mathbf{k}} \frac{1}{e^{\Delta(\mathbf{k}) / k_{B} T}+1}
$$

Using an ansatz of a formal similarity between a pure type II superconductor in high magnetic fields and a currentcarrying superconductor Maki 17 arrived at the following expression for the ratio $\alpha_{S} / \alpha_{N}$ between longitudinal attenuation in the mixed state and in the normal state:

$$
\frac{\alpha_{S}}{\alpha_{N}} \simeq 1-\frac{\Delta}{2 k_{B} T}
$$

where $\Delta^{2} \equiv\left\langle|\Delta(\mathbf{r})|^{2}\right\rangle$ is the real space average of the gap. As Maki used a semiclassical approximation he did only calculate the 0 'th harmonic. To compare with this result we expand Eq. (11) to first order in $\Delta(\mathbf{k})$. We get:

$$
\frac{\alpha_{S}}{\alpha_{N}}=1-\frac{\langle|\Delta(\mathbf{k})|\rangle_{\mathbf{k}}}{2 k_{B} T}
$$

where $\langle|\Delta(\mathbf{k})|\rangle_{\mathbf{k}}$ is the $\mathbf{k}$-space average of $|\Delta(\mathbf{k})|$. We see that our theory for the 0 'th harmonic, which is exact within the diagonal approximation produces a term linear in $\Delta$ for the ratio $\alpha_{S} / \alpha_{N}$ as does Maki's conjecture. This linear term is somewhat surprising as the Gor'kov expansion of the Green's functions would seem to imply that the first correction term is quadratic in $\Delta$. However, even in the zero field BCS-state one obtains $\mathbb{3}^{3} \alpha_{S} / \alpha_{N}=$ $2 /\left(\exp \left(\Delta / k_{B} T\right)+1\right)$ which cannot be expanded in $\Delta^{2}$. So our theory confirms Maki's ansatz of a linear correction term for the damping. Thus the non-perturbative linear term in the quasiparticle energy Eq.(6) coming essentially from the degeneracy of the Landau levels shows up in the attenuation result, making a calculation based on the Gor'kov expansion questipable. This is in contrast to the case of the expansion of the thermodynamic potential where contrary to earlier claims, 18 it can be proven that these non-perturbative terms cancel, thus making the Gor'kov expansion of the thermodynamic potential correct. 19 Our result substitutes Maki's real space average $\Delta=\left(\left\langle|\Delta(\mathbf{r})|^{2}\right\rangle\right)^{1 / 2}$ with the $\mathbf{k}$-space average of $|\Delta(\mathbf{k})|$.

For conventional superconductors in the Meissner state we know that the finite gap for all $\mathbf{k}$ supresses the attenuation by a factor $2 /\left(\exp \left(\Delta / k_{B} T+1\right)\right.$. We expect that the existence of gapless points will now change this result significantly. We therefore split the MBZ into two qualitatively different regions: the "gapped" region where we assume $\Delta(\mathbf{k})=$ $\langle\Delta(\mathbf{k})\rangle$ where $\langle\Delta(\mathbf{k})\rangle$ is the $\mathbf{k}$-space average of $\Delta(\mathbf{k})$, a the "gapless" region where we assume $\Delta(\mathbf{k})=\gamma k^{\eta}$. We furthermore assume that this latter fispersion law holds for all $\Delta(\mathbf{k}) \lesssim k_{B} T$. This is a slight generalization of the model used by Dukan and Tešanovićl in their theory for the dHvA oscillations. We assume that the gapped region takes up a fraction $\mathcal{F}$ of the MBZ. The contribution $\alpha(\mathbf{q}, \omega)_{0, \text { gap }}$ to the attenuation from the gapped region is then

$$
\alpha(\mathbf{q}, \omega)_{0, \text { gap }}=\frac{\omega^{2} m^{2}}{\hbar^{2} q h} \mathcal{F} \frac{2}{e^{\langle\Delta(\mathbf{k})\rangle / k_{B} T}+1}
$$

where $h=\hbar 2 \pi$. As expected the attenuation from the gapped part of the spectrum is strongly supressed due to the $2 /\left(\exp \left(\langle\Delta(\mathbf{k})\rangle / k_{B} T\right)+1\right)$ factor. This result for the 0 'th harmonic of the attenuation from the gapped part 
of the spectrum in the mixed state is the same as for the total attenuation in the Meissner state of a conventional superconductor. The qualitative new feature comes from the presence of the gapless points. Solving the 2-dimensional k-space integral in Eq.(11) using the assumed dispersion law, we obtain the contribution $\alpha(\mathbf{q}, \omega)_{g l}$ from the gapless part as

$$
\alpha(\mathbf{q}, \omega)_{0, g l}=Q_{g l} \frac{m \omega^{2}}{\hbar^{2} \omega_{c} q \pi}\left(\frac{k_{B} T}{\gamma}\right)^{2 / \eta} \frac{1-2^{1-2 / \eta}}{\eta} \Gamma\left(\frac{2}{\eta}\right) \zeta\left(\frac{2}{\eta}\right)
$$

where $Q_{g l}$ is the number of gapless points in the MBZ. Here $\zeta(x)$ is Riemann's zeta function and $\Gamma(x)$ is the gamma function. For a linear dispersion around the gapless points we especially obtain

$$
\alpha(\mathbf{q}, \omega)_{g l}=Q_{g l} \frac{m \omega^{2} \pi\left(k_{B} T\right)^{2}}{12 \hbar^{2} \omega_{c} q \gamma^{2}} .
$$

The relative size of the contributions from the gapped and gapless parts of the spectrum is determined by $\langle\Delta(\mathbf{k})\rangle / k_{B} T$, $\mathcal{F}$ and $\gamma$. For $\langle\Delta\rangle \gtrsim 3 k_{B} T$ the contribution from the gapped part can be ignored and the attenuation is given by Eq.(15). Since the normal state attenuation is given by $\alpha(\mathbf{q}, \omega)_{0, N}=m^{2} \omega^{2} / \hbar^{2} q h$ we get

$$
\frac{\alpha(\mathbf{q}, \omega)_{0, g l}}{\alpha(\mathbf{q}, \omega)_{0, N}}=Q_{g l} \frac{2 \hbar}{m \omega_{c}}\left(\frac{k_{B} T}{\gamma}\right)^{2 / \eta} \frac{1-2^{1-2 / \eta}}{\eta} \Gamma\left(\frac{2}{\eta}\right) \zeta\left(\frac{2}{\eta}\right) .
$$

This calculation is valid in the clean case where the momentum is conserved during the scattering process. As a first approximation to the dirty limit_ve can assume that there is no momentum conservation in the scattering process and take $k_{z}$ and $k_{z}^{\prime}$ as free variables 11 (i.e $k_{z}^{\prime} \neq k_{z}+q$ ). As in the Meissner state, this relaxation does not alter the result stated in Eq.(17). This is due to the fact we are looking at energies close to the Fermi level such that we can assume that the normal state density-of-states is constant. It has been shown 20 that for small impurity concentrations and weak scattering potentials the density of states behaves essentially in the same way as for the pure case. We therefore believe that our results are somewhat insensitive to the presence of impurities.

The Landau level structure of the normal state electron energies implies that there will oscillations in the attenuation as the external field varies. The oscillatory terms are given by the $j \neq 0$ terms in Eq.(10). We end up with the following expression for the first harmonic $\alpha(\mathbf{q}, \omega)_{g l, 1}$ of the attenuation from the gapless part of the spectrum:

$$
\begin{array}{r}
\alpha(\mathbf{q}, \omega)_{g l, 1}=Q_{g l} \frac{2 m \omega^{2}}{\hbar k_{B} T q \pi} \cos \left(2 \pi\left(n_{f}-\epsilon\left(k_{z}^{*}\right) / \hbar \omega_{c}\right)\right) \int_{0}^{\infty} k d k \int_{0}^{\infty} d x \\
\times\left(e^{-\sqrt{x^{2} \hbar^{2} \omega_{c}^{2}+\gamma^{2} k^{2 \eta}} / k_{B} T}+1\right)^{-1}\left(e^{\sqrt{x^{2} \hbar^{2} \omega_{c}^{2}+\gamma^{2} k^{2 \eta}} / k_{B} T}+1\right)^{-1} \frac{\cos (2 \pi x) x \omega_{c}}{\sqrt{x^{2} \hbar^{2} \omega_{c}^{2}+\gamma^{2} k^{2 \eta}}}
\end{array}
$$

This integral can be solved in the case of a linear spectrum around the gapless points (i.e $\eta=1$ ). In this case we obtain for the 1st harmonic of the attenuation

$$
\begin{aligned}
\alpha(\mathbf{q}, \omega)_{g l, 1}= & Q_{g l} \frac{m \omega^{2}}{q \hbar^{2} \omega_{c} \pi}\left(\frac{k_{B} T}{\gamma}\right)^{2} \cos \left(2 \pi\left(n_{f}-\epsilon\left(k_{z}^{*}\right) / \hbar \omega_{c}\right)\right) \\
& \times\left[\frac{\pi^{2} \cosh \left(2 \pi^{2} k_{B} T / \hbar \omega_{c}\right)}{\sinh ^{2}\left(2 \pi^{2} k_{B} T / \hbar \omega_{c}\right)}-\frac{1}{\left(2 \pi k_{B} T / \hbar \omega_{c}\right)^{2}}\right] .
\end{aligned}
$$

For low temperatures this goes as $T^{2}$. In the case of a general dispersion law around the gapless point given by $\Delta(\mathbf{k})=\gamma k^{\eta}$, the leading term for the 1 st harmonic goes as $(T / \gamma)^{2 / \eta}$. This should be contrasted to the Meissner state, or the contribution from the gapped part of the spectrum, where the oscillatory terms will again be damped by a factor $2 /\left(\exp \left(\langle\Delta(\mathbf{k})\rangle / k_{B} T\right)+1\right)$. In the case of the normal state, the 1st harmonic is

$$
\alpha(\mathbf{q}, \omega)_{N, 1}=\frac{m^{2} \omega^{2}}{h q \hbar^{3} \omega_{c}} \frac{4 k_{B} T \pi^{2}}{\sinh \left(2 \pi^{2} k_{B} T / \hbar \omega_{c}\right)} \cos \left(2 \pi\left(n_{f}-\epsilon\left(k_{z}^{*}\right) / \hbar \omega_{c}\right)\right)
$$

which is independent of $T$ for low temperatures. In the case of a dispersion law along the $z$-direction given by $\epsilon\left(k_{z}\right)=$ $t \cos \left(k_{z} a_{z}\right)$ we have to substitute $m / q$ by $2 / t a_{z}\left|\sin \left(k_{z}^{*} a_{z}\right)-\sin \left(\left(k_{z}^{*}+q\right) a_{z}\right)\right|$ in Eq. (15) and $m \cos \left(2 \pi\left(n_{f}-\epsilon\left(k_{z}^{*}\right) / \hbar \omega_{c}\right)\right) / q$ by $\sum_{i=1,2} \cos \left(2 \pi\left(n_{f}-\epsilon\left(k_{z, i}^{*}\right) / \hbar \omega_{c}\right)\right) / t a_{z}\left|\sin \left(k_{z}^{*} a_{z}\right)-\sin \left(\left(k_{z}^{*}+q\right) a_{z}\right)\right|$ in Eq.(19)-(20) where $k_{z, i}$ are the two solutions of the equation $t \cos \left(\left(k_{z}+q\right) a_{z}\right)-t \cos \left(k_{z} a_{z}\right)=\hbar \omega$. 
In Fig. 2 we show an example of the acoustic attenuation calculated numerically for two different coupling strengths as a function of $n_{f}$. We have solved the BdG equations self-consistently in $3 \mathrm{D}$ as a function of the external magnetic field at constant chemical potential. In this paper we have chosen to work with a constant chemical potential. The difference between holding the chemical potential constant and holding the number of particles constant is negligible in 3D for the normal state.12 Even in 2D it can be shown that the superconducting order tends to suppress the difference between the two cases in the mixed state. 6 The attenuation is calculated via Eq.(5). We have chosen parameters such that $\omega_{D} / \omega_{c}=5, k_{B} T / \hbar \omega_{c}=0.05$ and $\omega / \omega_{c}=0.01$ when $n_{f}=12$, where $\omega_{D}$ is the usual cutoff of the pairing interaction around the Fermi surface. The solid curve is the normal state attenuation which is continued into the mixed state to facilitate comparison with the mixed state attenuation; the dashed curve corresponds to the coupling strength $g / \hbar \omega_{c} l^{3}=7.85$ while the dash-dot curve corresponds to $g / \hbar \omega_{c} l^{3}=8.7$. The dispersion law along the $z$-direction is $\epsilon\left(k_{z}\right)=t \cos \left(k_{z} a_{z}\right)$ where $t / \hbar \omega_{c}=0.4$. In Fig. 3 we have plotted the corresponding order-parameter which in the lowest Landau level approximation for the pairing (which is valid close to $H_{c 2}$ ) 1 can be characterized by the dimensionless number $\Delta_{0} \propto \Delta(\mathbf{k})$. The connection between $\Delta_{0}$ and $\Delta(\mathbf{k})$ or $\Delta(\mathbf{r})$ is given by an obvious generalisation to $3 \mathrm{D}$ of the results by Norman et al. $\mathrm{B}$ The phase transition between the normal and the mixed state occurs for $n_{f} \simeq 7.6$ with $g / \hbar \omega_{c} l^{3}=8.7$ and for $n_{f} \simeq 9.5$ with $g / \hbar \omega_{c} l^{3}=7.85$. As can be seen from Fig. 2 , the oscillations of the attenuation due to the Landau level quantization persist into the mixed state, although they are damped as compared to the normal state oscillations. Eventually the oscillations die out when the off-diagonal pairing becomes dominant and the diagonal approximation breakes down. This happens for $n_{f} \gtrsim 11$ with $g / \hbar \omega_{c} l^{3}=8.7$. Comparing the attenuation for the two coupling strengths in the region $10 \leq n_{f} \leq 11$ we get $\alpha_{0}(7.85) / \alpha_{0}(8.7) \simeq 1.9$ and $\alpha_{1}(7.85) / \alpha_{1}(8.7) \simeq 24$ where $\alpha(g)_{i}$ is the $i$ 'th harmonic of the attenuation for the coupling strength $g$. Since $\langle\Delta(\mathbf{k})\rangle$ can be calculated 3 directly from $\Delta_{0}$, and the coefficient $\gamma$ in the dispersion law around the gapless points is proportional to $g \Delta_{0}$, we can compare the numerical results with the analytical predictions outlined above. If the quasiparticle spectrum is essentially gapped, Eq.(14) predicts that $\alpha_{0}(7.85) / \alpha_{0}(8.7)=f\left(\left\langle\Delta_{7.85}\right\rangle\right) / f\left(\left\langle\Delta_{8.7}\right\rangle\right) \simeq 8.8$. If the attenuation is primarily originating from gapless points in the quasiparticle spectrum, Eq.(15) predicts that $\alpha_{0}(7.85) / \alpha_{0}(8.7)=\left(8.7 \Delta_{0}(8.7) / 7.85 \Delta_{0}(7.85)\right)^{2 / \eta}$. This gives 2.5 for $\eta=1$ and 1.6 for $\eta=2$. Hence the numerical calculation $\left(\alpha_{0}(7.85) / \alpha_{0}(8.7) \simeq 1.9\right)$ imply (i) that the gapless points dominate the attenuation in agreement with our analytical results and (ii) that the dispersion law is somewhere between $\eta=1$ and $\eta=2$, since the gapless predictions agree reasonably well with the numerical results while the gapped predictions are qualitatively wrong. We cannot however make a quantitative numerical determination of the dispersion law around the gapless points. This is due to the fact that in order to reduce the computation load, which is high in this $3 \mathrm{D}$ case, we have to choose a k-mesh with a rather large spacing between the points. (the mesh consists of $100 \times 50$ points) This means that the gapless regions in the quasiparticle spectrum are only probed by a few $\mathbf{k}$-vectors, thereby prohibiting a quantitative determination of the dispersion law. Likewise, the analysis above predicts that $\alpha_{1}(7.85) / \alpha_{1}(8.7)$ equals 2.5 and 1.6 for $\eta=1$ and $\eta=2$ respectively. The $\eta=1$ prediction of 2.5 agrees well with the numerical result $\alpha_{1}(7.85) / \alpha_{1}(8.7) \simeq 2.4$. Again, any quantitative comparison would require a much finer $\mathbf{k}$-mesh. A further complication is that due to the number of Landau levels participating in the pairing (approx. 10), the oscillations in the attenuation are quickly damped and the diagonal approximation is only valid over relatively few oscillations. This problem could be avoided if we were to perform the calculations for experimentally realistic parameters, where there are many more Landau levels in the pairing region; however we have not been able to run the programs for such parameters due to the intense computation load. The above example does show however that the numerical calculations support our analytic theory. In short, the normal state oscillations in the attenuation continue into the mixed state and the damping is dominated by gapless points not too far into the mixed state.

Hence we have calculated the 0'th and the 1st harmonic of the acoustic attenuation in the mixed state. The presence of gapless points enhances the acoustic attenuation above the conventional value for the Meissner state. When $\langle\Delta\rangle \gtrsim 3 k_{B} T$ such that we can ignore the contribution from the gapped part of the spectrum, the temperature dependence of the attenuation is a power law given by $\alpha \propto T^{2 / \eta}$. Furthermore, we predict that one should observe oscillations in the signal as the external field is varied. The magnitude of these oscillations should have the same temperature dependence as the average value of the signal. Hence, by looking at the temperature dependence of the attenuation one should be able to detect the presence of the gapless points and to extract the dispersion law around these points. From the diagonal approximation it follows that $\gamma \propto\langle\Delta\rangle$ where $\langle\Delta\rangle$ is the $\mathbf{k}$-space (or real space) average of the gap. Hence close to the upper critical field $H_{c 2}$ we expect $\gamma \propto \sqrt{1-H / H_{c 2}}$. This would mean that $\alpha(\mathbf{q}, \omega) \propto\left(1-H / H_{c 2}\right)^{-1 / \eta}$ close to $H_{c 2}$. 


\section{LOW TEMPERATURE}

We will now consider the limit where $k_{B} T \ll \hbar \omega$. In this limit only the third delta function in Eq.(5)), which describes the creation of two quasiparticles will contribute to the damping. We first calculate the 0'th harmonic of the attenuation. Using the Poisson identity, making a substitution of variables and using the fact that $v_{s} \equiv \omega / q \ll v_{f}$ ( $v_{f}$ is the Fermi velocity) we obtain the 0 'th harmonic of the attenuation, when $\epsilon\left(k_{z}\right)=k_{z}^{2} / 2 m$, as

$$
\alpha(\mathbf{q}, \omega)_{0}=\frac{m \omega}{q \hbar^{3} \omega_{c} L_{x} L_{y}} \sum_{\mathbf{k}} \int_{\Delta(\mathbf{k})}^{\omega-\Delta(\mathbf{k})} d E[1-f(E)-f(\omega-E)] \frac{E(\omega-E)+\Delta^{2}}{\sqrt{\left(E^{2}-\Delta^{2}\right)\left((\omega-E)^{2}-\Delta^{2}\right)}} .
$$

In the limit $\omega / k_{B} T \rightarrow \infty$ this integral can be written as a complete elliptic integral21 and we obtain

$$
\alpha(\mathbf{q}, \omega)_{0}=\frac{m \omega^{2}}{4 \pi^{2} \hbar^{2} \omega_{c} q} \int d^{2} \mathbf{k} \mathcal{E}\left(\sqrt{1-4 \Delta(\mathbf{k})^{2} / \hbar^{2} \omega^{2}}\right) .
$$

Here $\mathcal{E}(k)$ is the complete elliptic integral of the second kind. The existence of the gapless points again gives rise to a qualitatively different result for the ultrasonic attenuation in the mixed state as compared to the Meissner state. This is most easily understood by the observation that there will always be attenuation for any frequency in the mixed state since there are always quasiparticle states with $\Delta(\mathbf{k}) \leq \hbar \omega / 2$. Hence the phonon will always have enough energy to create two quasiparticles. This is in contrast to the Meissner state, where there is no attenuation for $\hbar \omega<2 \Delta$. 21 Thus a direct experimental signature of these gapless points would be the absence of the discontinuity in the attenuation which is present in the Meissner state21, when $\hbar \omega=2 \Delta$, and the presence of acoustic attenuation in the mixed state as $\omega \rightarrow 0$. We again assume that the dispersion law around the gapless points to leading order is given by $\Delta(\mathbf{k})=\gamma k^{\eta}$ $(k=|\mathbf{k}|)$ in the region that contributes to the attenuation (i.e for $\Delta(\mathbf{k}) \leq \hbar \omega / 2)$. Using this we obtain

$$
\alpha(\mathbf{q}, \omega)_{0}=Q_{g l} \frac{m \omega^{2 / \eta+2}}{2 \pi \hbar^{2} \omega_{c} q(2 \gamma)^{2 / \eta}} \int_{0}^{1} x d x \mathcal{E}\left(\sqrt{1-x^{2 \eta}}\right)=n \frac{m \omega^{2 / \eta+2}}{2 \pi \hbar^{2} \omega_{c} q(2 \gamma)^{2 / \eta}} I_{\eta} .
$$

Since the attenuation for the normal state is $\alpha(\mathbf{q}, \omega)_{0, N}=m^{2} \omega^{2} / 2 \pi \hbar^{3} q$ we have

$$
\frac{\alpha(\mathbf{q}, \omega)_{0}}{\alpha(\mathbf{q}, \omega)_{0, N}}=Q_{g l}\left(\frac{\omega}{2 \gamma}\right)^{2 / \eta} \frac{\hbar I_{\eta}}{m \omega_{c}}
$$

For the same reasons as for the $\hbar \omega \ll k_{B} T$ case, we expect Eq.(24) to also be valid in the dirty limit. The remaining integral in Eq. (23) can be solved for various $\eta$. We obtain, for instance, $I_{1}=2 / 3, I_{2}=\pi^{2} / 16, I_{1 / 2}=32 / 45$ etc. Again the attenuation has an oscillatory behaviour as a function of the magnetic field $H^{-1}$. From the Poisson identity we get for the 1st harmonic

$$
\begin{array}{r}
\alpha(\mathbf{q}, \omega)_{1}=Q_{g l} \frac{m \omega}{4 \hbar^{3} \omega_{c} q 2 \pi} \sum_{j=-1,1} e^{2 \pi i j\left(n_{f}-\epsilon\left(k_{z}^{*}\right) / \hbar \omega_{c}\right)} \int k d k \int d \xi d \xi^{\prime} \delta\left(E+E^{\prime}-\omega\right) \\
\times\left[(1-\xi / E)\left(1+\xi^{\prime} / E^{\prime}\right)+\frac{\Delta(k)^{2}}{E E^{\prime}}\right] e^{i j \xi / \hbar \omega_{c}} .
\end{array}
$$

We have not been able to solve this integral exactly. However, in the region where the diagonal approximation holds (i.e for $\left.\omega \lesssim \hbar \omega_{c} / 4\right)$ one can expand the factor $e^{i l \xi / \hbar \omega_{c}}$ to a good approximation. This immediately yields that, to leading order in $\omega / \omega_{c}$, the amplitude of the first harmonic varies as $\omega^{2 / \eta+2}$. The next correction term will go as $\omega^{2 / \eta+4}$.

So we see that the existence of the gapless points in the MBZ implies that there is a finite attenuation for any frequency of the sound wave. There will be no discontinuity in the attenuation as a function of the sound wave frequency. As the external field is varied one should observe oscillations in the attenuation. The dependence of the attenuation on frequency is algebraic and the power-law is determined by the dispersion law around the gapless points. If $\Delta(\mathbf{k})=\gamma k^{\eta}$ we obtain $\alpha \propto \omega^{2 / \eta+2}$. The absence of the discontinuity and the frequency dependence of the attenuation should in principle provide the possibility of experimentally determining the existence and dispersion law for the gapless points. Again we expect $\alpha(\mathbf{q}, \omega) \propto\left(1-H / H_{c 2}\right)^{-1 / \eta}$ close to $H_{c 2}$. By making the same substitutions as in the $\hbar \omega \ll k_{B} T$ limit, one can obtain the results for the case $\epsilon\left(k_{z}\right)=t \cos \left(k_{z} a_{z}\right)$ relevant for layered structures. 


\section{CONCLUSION}

In this paper we have considered the acoustic attenuation in a type II BCS superconductor at high magnetic fields using both numerical and analytical methods. We have shown that away from the semiclassical regime where the Landau level structure of the electronic states is important, the attenuation will in general be an oscillatory function of the external magnetic field. Furthermore, since the attenuation probes the quasiparticle density of states, the presence of gapless points in the quasiparticle spectrum makes the attenuation qualitatively different as compared to the Meissner state attenuation. For $k_{B} T \ll \hbar \omega_{c}$ the attenuation is an algebraic function of the frequency and there is no discontinuity as opposed to the Meissner state attenuation. For $k_{B} T \gg \hbar \omega_{c}$ the attenuation is an algebraic function of the temperature. The exponent of the power law is determined by the dispersion law around the gapless points. This behaviour should in principle be experimentally detectable; such an experiment would provide confirmation of the existence and nature of the gapless points.

\section{ACKNOWLEDGEMENTS}

This work has been supported in part by EPSRC grant GR/K 15619 (VNN and NFJ) and by The Danish Research Academy (GMB).

${ }^{1}$ M. Rasolt and Z. Tešanović, Rev. Mod. Phys. 64, 709 (1992)

${ }^{2}$ J.E. Graebner and M. Robbins, Phys. Rev. Lett. 36, 422 (1976)

${ }^{3}$ See M.R. Norman, A.H. MacDonald, and H. Akera, Phys. Rev. B 51, 5927 (1995) and references therein.

${ }^{4}$ S. Dukan and Z. Tešanović, Phys. Rev. B 49, 13017 (1994)

${ }^{5}$ S. Dukan and Z. Tešanović, Phys. Rev. Lett 74, 2311 (1995)

${ }^{6}$ G.M. Bruun, V.N. Nicopoulos and N.F. Johnson, Phys. Rev. B 56, 809 (1997)

${ }^{7}$ K. Maki, in Superconductivity, R.D. Parks (ed) (Marcel Dekker, New York, 1969)

${ }^{8}$ K. Scharnberg, J. Low Temp. Phys. 6, 51 (1972)

${ }^{9}$ C. Caroli and K. Maki, Phys. Rev. 159, 316 (1967)

${ }^{10}$ D. Dominguez, L. Bulaevskii, B. Ivlev, M. Maley, and A. R. Bishop, Phys. Rev. B 53, 6682 (1996)

${ }^{11}$ G. D. Mahan, Many particle physics (Plenum, New York, 1981)

12 D. Schoenberg, Magnetic Oscillations in Metals (Cambridge University Press, Cambridge, England, 1984)

13 J.R. Schrieffer, Theory of Superconductivity (W.A.Benjamin, New York, 1964)

${ }^{14}$ P.G. de Gennes, Superconductivity of Metals and Alloys (Addison-Wesley, New York, 1989)

${ }^{15}$ H. Akera, A.H. MacDonald, S.M. Girvin, and M.R. Norman, Phys. Rev. Lett. 67, 2375 (1991); S. Dukan, A.V. Andreev and Z. Tešanović, Physica C 183, 355 (1991); J.C. Ryan and A.K. Rajagopal, Phys. Rev. B 47, 8843 (1993); V.N. Nicopoulos and P. Kumar, Phys. Rev. B 44, 12080 (1991);

${ }^{16}$ M. R. Norman and A. H. MacDonald, Phys. Rev. B 54, 4239 (1996)

17 M. Cyrot and K. Maki Phys. Rev. 156, 433 (1967); K. Maki, Phys. Rev. 156, 437 (1967)

18 S. R. Bahcall, Solid State Commun. 100, 297 (1996)

${ }^{19}$ G. M. Bruun and V. N. Nicopoulos, J. Phys. Condens. Matter 9, 2773 (1997)

${ }^{20}$ S. Dukan and Z. Tešanović, Phys. Rev. B 56, 838 (1997)

${ }^{21}$ V. M. Bobetic, Phys. Rev. 136, A1535 (1964) 


\section{Figure Captions}

Fig. 1: The lowest quasiparticle energy in units of $\hbar \omega_{c}$ for two different values of the order parameter $\left(\langle\Delta(\mathbf{k})\rangle \simeq 0.3 \hbar \omega_{c}\right.$ and $\left.\langle\Delta(\mathbf{k})\rangle \simeq 0.05 \hbar \omega_{c}\right)$. The $\mathbf{k}$-vector is measured in units of $2 \pi / L_{x}$ where $L_{x}$ is the size of the sample in the $x$-direction. The solid lines show the exact numerical result while the dashed lines correspond to the diagonal approximation.

Fig. 2: The attenuation as a function of $n_{f}$. The solid line is the normal state attenuation. The dashed line is for the coupling $g / \hbar \omega_{c} l^{3}=7.85$. The dash-dot line is for $g / \hbar \omega_{c} l^{3}=8.7$.

Fig. 3: The order-parameter as a function of $n_{f}$. The dashed line is for the coupling $g / \hbar \omega_{c} l^{3}=7.85$ while the dash-dot line is for $g / \hbar \omega_{c} l^{3}=8.7$. 
Figure 1 (Bruun, prb)

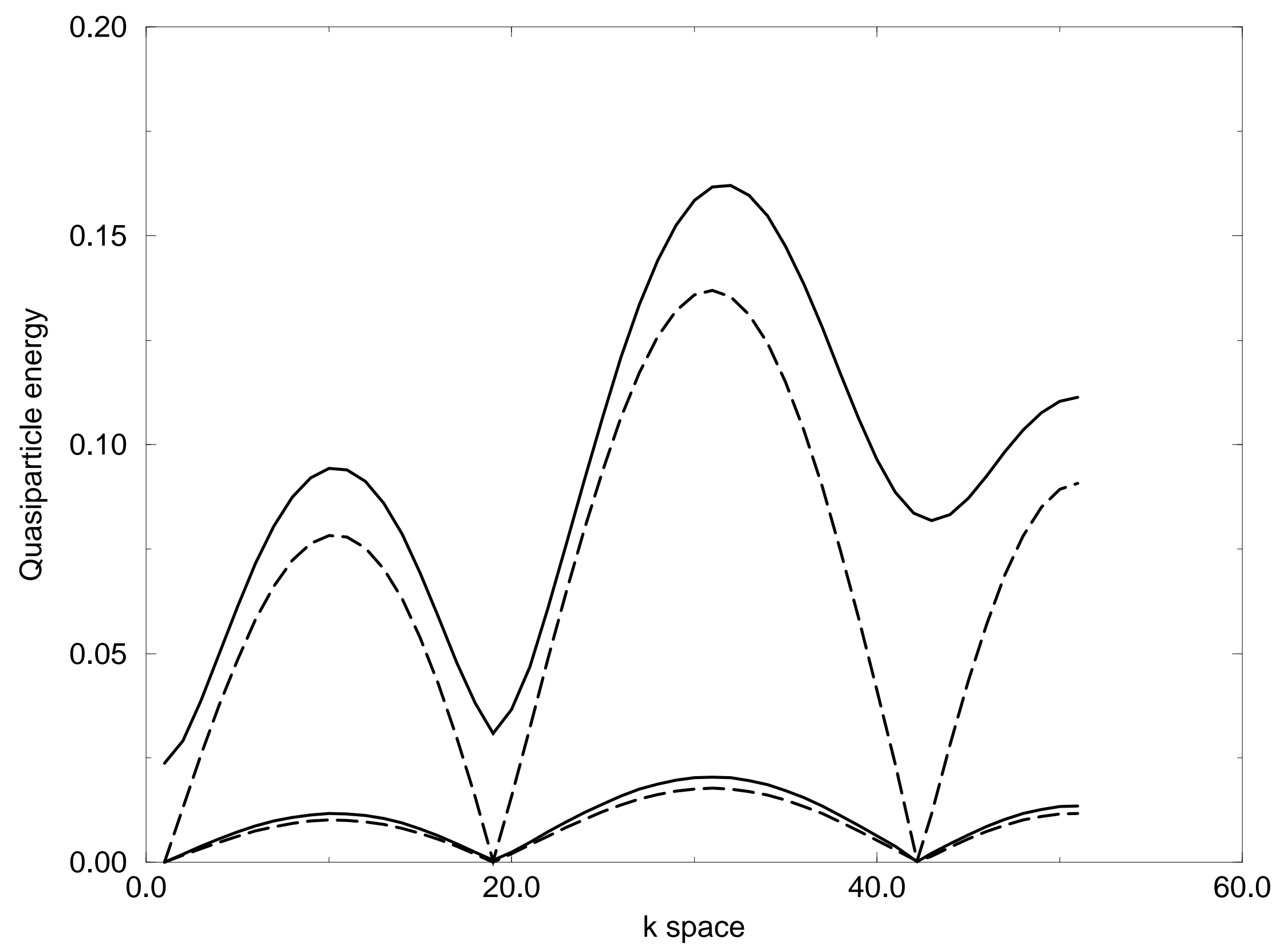


Figure 2 (Bruun, prb)

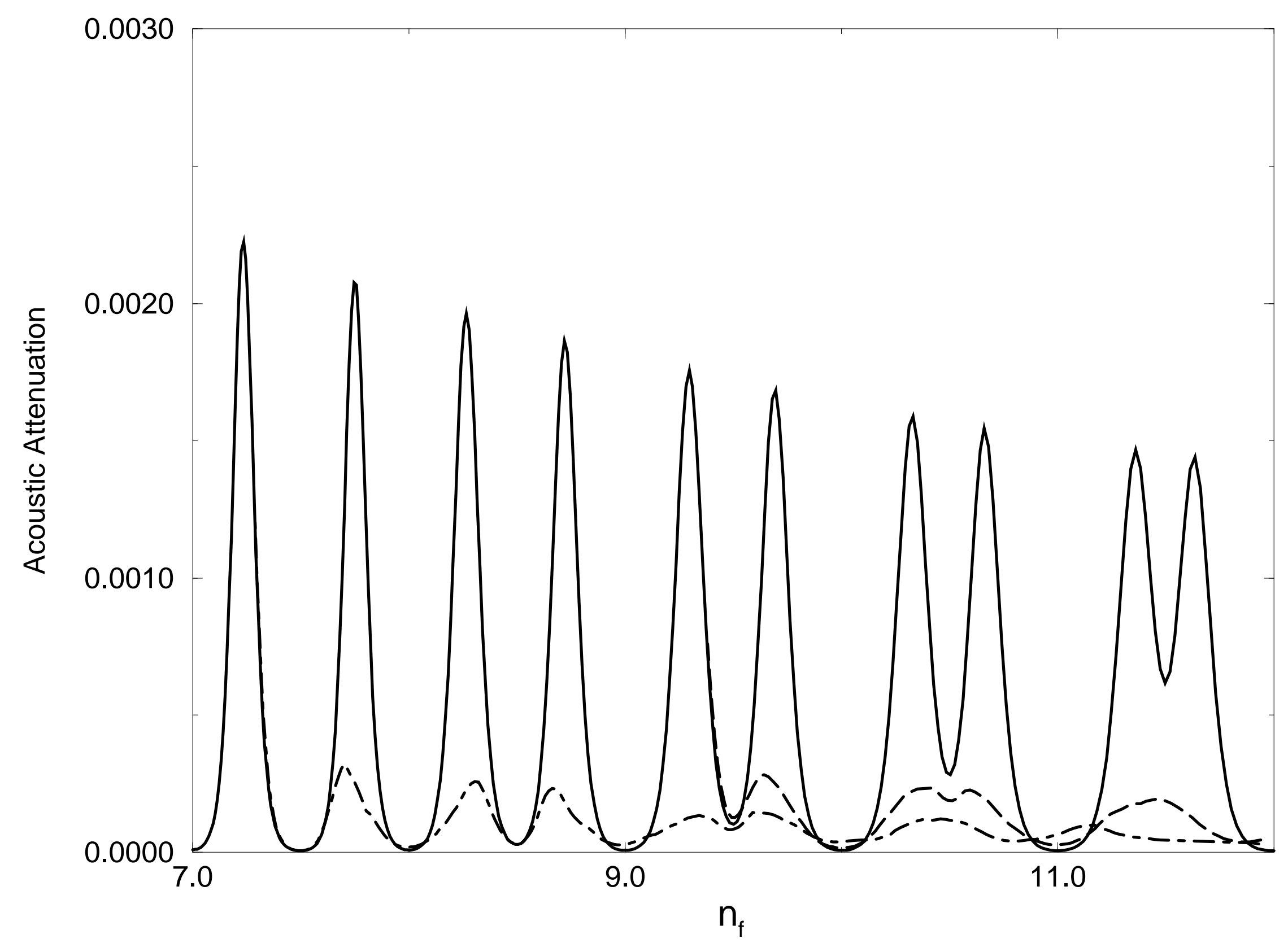


Figure 3 (Bruun, prb)

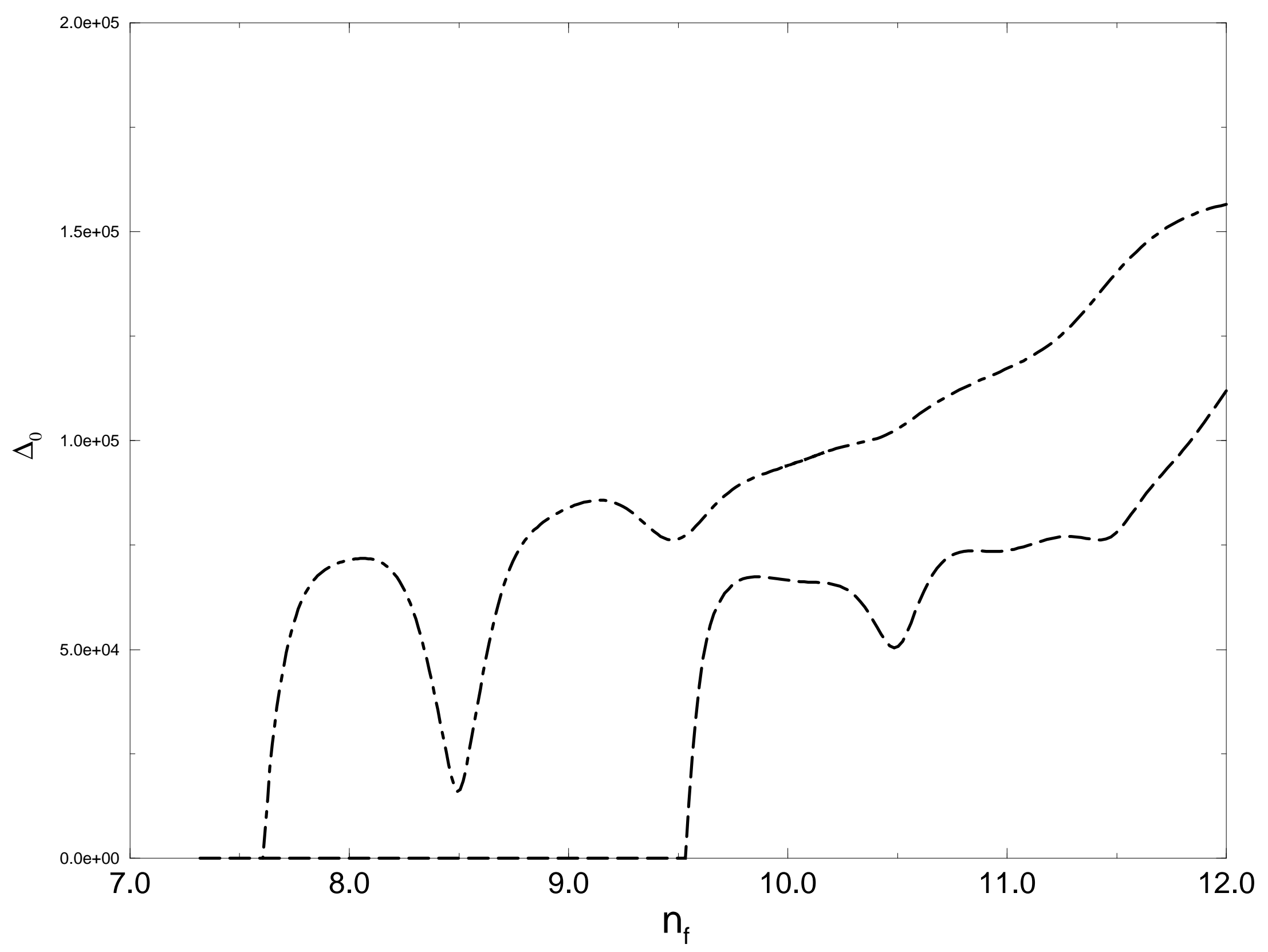

